\title{
Plasma: An International Open Access Journal for All of Plasma Science
}

\author{
David A. Gates \\ Princeton Plasma Physics Laboratory, Princeton University, Princeton, NJ 08543, USA; dgates@pppl.gov
}

Received: 3 January 2018; Accepted: 8 January 2018; Published: 12 January 2018

Plasma is an open access, cross-disciplinary scholarly journal of scientific studies related to all aspects of plasma science, such as plasma physics, plasma chemistry and space plasma. It publishes reviews, research articles, short communications and letters. Topics also include experimental and theoretical results, and progress in interdisciplinary aspects and applications of relevance to plasmas. Our aim is to encourage scientists to publish their experimental and theoretical results in as much detail as possible so that they can be reproduced. The broad set of topics covered include: electron, ion, and plasma sources, basic plasma phenomena, plasma theory and modeling, nonlinear plasma dynamics, dusty plasmas (complex plasmas): theory and experiment, complex and nonlinear phenomena, nonlinear waves, instabilities, turbulence, microwave/plasma interaction, laser-plasma interactions, magnetically confined plasma, low temperature plasma, high temperature plasma, astrophysics and space plasma, magnetic reconnection and laboratory astrophysics, plasma-based technology and treatments, plasma diagnostics, plasma chemistry and processing, plasma for controlled fusion research, industrial/commercial applications of plasma and plasma flow control.

Plasma as a physical system per se was first observed in 1879 by Sir William Crookes, who described it as "radiant matter". The name "Plasma" was coined by the Nobel prize winning chemist, Irving Langmuir, in 1927. Langmuir may be acknowledged as the world's first plasma physicist for his discovery of the plasma sheath phenomenon. The genesis of plasma physics at the hands of a famous chemist foreshadowed the eventual state of plasma science that exists today. Plasmas are studied by: material scientists, fusion energy scientists, solar scientists, astrophysicists, physical chemists, telecommunications researchers, and many others.

Because of the wide range of applications for plasma science, there are a correspondingly large number of theoretical models. Additionally, the wide range of temporal and spatial scales (for example, in magnetized plasmas length scales can vary from the electron gyro-radius $\left(<10^{-3} \mathrm{~m}\right)$ to galactic scales) that can affect plasma behavior means that there is also a hierarchy of models that differentiate on scale.

The broad interest in plasmas is a reflection of the fact that plasma makes up most of the visible universe and the important role that this state of matter plays in processes that impact our daily lives. For example, the phenomenon of magnetic reconnection in solar flares can cause large-scale coronal mass ejections (CME). When these CME's impinge on the Earth's magnetic field, they can inflict widespread havoc on Earth-based electrical systems. Similar in impact, the field of plasma processing has developed new material coating and micro-chip fabrication techniques that have changed the way humans live.

Given this diversity of applications, the need for a comprehensive journal spanning all of plasma science is evident. This journal's goal is to provide a central repository of high quality, peer-reviewed articles that can disseminate information among the members of this varied research community. All relevant high quality, innovative, plasma science papers are welcome and, following a rigorous review procedure, will be published-if accepted-in as short time as possible. 
The Editorial Board is comprised of a diverse group of experts that reflects the broad range of topics spanned by the journal and the varied interests of the research community it represents. The success of this endeavor depends on the journal's ability to convince this community of the value of this contribution. In turn, the value of the contribution will depend on the high quality articles and reviews that I encourage you and your colleagues to submit to Plasma. I, and the other members of the Editorial Board look forward to your contributions.

(C) 2018 by the author. Licensee MDPI, Basel, Switzerland. This article is an open access article distributed under the terms and conditions of the Creative Commons Attribution (CC BY) license (http://creativecommons.org/licenses/by/4.0/). 